\title{
Dendritic cell-tumor cell hybrids enhance the induction of cytotoxic $T$ lymphocytes against murine colon cancer: A comparative analysis of antigen loading methods for the vaccination of immunotherapeutic dendritic cells
}

\author{
TAKASHI YASUDA, TAKASHI KAMIGAKI, TETSU NAKAMURA, TATSUYA IMANISHI, SHUN HAYASHI, \\ KENTARO KAWASAKI, SHIRO TAKASE, TETSUO AJIKI and YOSHIKAZU KURODA
}

Department of Gastroenterological Surgery, Graduate School of Medical Sciences, Kobe University, Kobe 650-0017, Japan

Received July 18, 2006; Accepted August 23, 2006

\begin{abstract}
Dendritic cells (DCs) have been used successfully for inducing effective anti-tumor immune responses in advanced cancer patients undergoing tumor-specific immunotherapy. Appropriate antigen pulsing is a crucial parameter for optimizing the efficacy of immunotherapy as well as antitumor protection therapy. Using a murine colon cancer model, we evaluated the anti-tumor efficacy of four different preparations of DC vaccines that contained either a whole tumor or its derivatives, including i) DCs pulsed with tumor lysate, ii) DCs pulsed with necrotic tumor cells, iii) DCs pulsed with apoptotic tumor cells, and iv) DC-tumor cell fusion hybrids. Our data show that DC-tumor cell fusion hybrids and DCs pulsed with irradiated apoptotic tumor cells were more potent than DCs with freeze-thawed necrotic tumor cells for the induction of protective anti-tumor responses. The vaccination of DCs pulsed with tumor lysate failed to elicit any anti-tumor effect. In animals administered with higher doses of a tumor-cell challenge, DC-tumor cell fusion hybrids elicited the most effective anti-tumor response. Among the preparations tested, mice immunized with DCtumor cell fusion hybrids resulted in the greatest induction of cytotoxicity as measured by the cytotoxic $\mathrm{T}$ lymphocyte activity of both the splenocytes and the Thy1.2-positive $\mathrm{T}$ lymphocytes. Furthermore, the in vitro production of IFN- $\gamma$ polarized to the Th1 cytokine responses was highest in the splenocytes derived from mice vaccinated with DC-tumor
\end{abstract}

Correspondence to: Dr Takashi Kamigaki, Department of Gastroenterological Surgery, Graduate School of Medical Sciences, Kobe University, Kusunoki-cho 7-5-1, Chuo-ku, Kobe 650-0017, Japan

E-mail: tkamy@med.kobe-u.ac.jp

Abbreviations: DC, dendritic cell; TC, tumor cell; APC, antigenpresenting cell; FSC, forward scatter; SSC, side scatter

Key words: dendritic cell-based immunotherapy, antigen loading, cytotoxic T lymphocytes, dendritic cell-tumor cell hybrids, tumor immunity cell fusion hybrids. Our results suggest that DC-tumor cell fusion hybrids are more potent inducers of protection against solid tumors, such as colon cancer, than other antigenloading strategies using whole tumor cell materials.

\section{Introduction}

Dendritic cells (DCs) are the most potent antigen-presenting cells (APCs) and are essential for the initiation and maintenance of immune responses (1). By contributing to the generation and proliferation of tumor-specific cytotoxic $\mathrm{T}$ lymphocytes (CTLs) and helper T cells (Th cells), DCs play a critical role in the induction of protective and therapeutic anti-tumor immunity. Numerous strategies for inducing efficient anti-tumor immunity with tumor antigen (Ag)loaded DCs have been reported in murine tumor models (2-5) and in trials in tumor-bearing patients (6-9). Optimization of the treatment parameters, including the DC source, DC maturation, choice of antigens (Ags), Ag-loading method, sites of injection, dosing, and appropriate anti-tumor immunological monitoring, will be required to further improve the efficacy of this approach.

There are two general approaches for preparing and loading tumor Ags: One uses a single tumor-associated Ag for the induction of a single antigen-specific monoclonal CTL, and the other uses whole tumor cells or their derivatives for the induction of multiple antigen-specific polyclonal CTLs. Variations in the former approach include the use of DCs pulsed with MHC class I-restricted tumor Ag-derived peptides $(10,11)$, DCs transfected with cDNA encoding a particular tumor-associated Ag (12), and tumor-peptide pulsed DC-derived exosomes (13). Although clinical trials using peptide-pulsed DCs have demonstrated immunological and clinical responses in cancers such as malignant melanoma $(7,14)$ and prostate cancer $(8)$, the application of specific peptides is limited to patients who express class I MHCs restricted to peptides associated with those tumors. Peptidebased immunotherapies also fail to utilize MHC class II and CD40L-mediated $\mathrm{CD}^{+} \mathrm{T}$ cell helper responses.

Various methods have been reported for generating DCbased vaccines using whole tumor cells or their derivatives including DCs pulsed with tumor-associated native or recom- 
binant proteins or DCs transfected with whole tumor cellderived RNA (15). In clinical trials, some investigators reported the efficacy of DCs pulsed with whole tumor lysates $(3,7)$ or stressed tumor cells (necrotic or apoptotic) $(16,17)$, which are more practical to obtain than isolated proteins or RNA.

DC-tumor cell fusion hybrids (DC/TC hybrids) have been shown to demonstrate superior efficacy for the treatment of murine tumor models (18-21). It is important that the hybrids express multiple tumor antigens in the context of MHC class I and/or class II molecules as well as co-stimulatory molecules essential for T-cell activation. Moreover, DC/TC hybrids have been shown to be efficacious for the treatment of both melanoma and renal cell carcinoma in Phase I/II clinical trials $(22,23)$. Strict comparative analyses that optimize methods for Ag-loading whole tumor cells or their derivatives have been conducted for melanoma, thymoma or acute myeloid leukemia (19-21).

In the present study, we compared the efficacy of four different strategies for pulsing DCs with whole tumor cell antigens on anti-tumor immunity in a murine colon carcinoma model. These strategies included DCs pulsed with i) tumor lysate, ii) freeze-thawed necrotic tumor cells, iii) irradiated apoptotic tumor cells, and iv) DC/TC hybrids. We also compared the potency of apoptotic tumor cells relative to necrotic tumor cells for use in the preparation of stressed tumor cells. The potency of CTL responses, the Th1/Th2 cytokine balance, and the in vivo anti-tumor immunity against the tumor challenge was also evaluated.

\section{Materials and methods}

Mice. Female Balb/c (H-2 $\left.{ }^{\mathrm{d}}\right)$ mice aged 6-8 weeks, purchased from CLEA JAPAN, Inc. (Tokyo, Japan), were housed in a specific pathogen-free environment at the Animal Maintenance Facility at Kobe University of Medicine. All mice were used at 8-12 weeks of age.

Cell lines. The murine colon adenocarcinoma cell line Balb/c-derived CT26 was purchased from ATCC (Rockville, MD, USA) and maintained in a complete RPMI-1640 tissue culture medium supplemented with $10 \%$ fetal bovine serum, L-glutamine, $100 \mu \mathrm{g} / \mathrm{ml}$ streptomycin and 100 units $/ \mathrm{ml}$ penicillin at $37{ }^{\circ} \mathrm{C}$ in a humidified atmosphere of $5 \% \mathrm{CO}_{2}$.

Dendritic cells. Dendritic cells derived from Balb/c mouse bone marrow (BM-DCs) were generated as described previously (24). Briefly, bone marrow was harvested from mouse femurs and tibiae and filtered through a 100-mm nylon cell strainer (BD Biosciences-Labware, San Jose, CA, USA). Whole bone-marrow cells were added to 24-well plates (BD Biosciences-Labware) at $1 \times 10^{6}$ cells/ml RPMI-1640 supplemented with $10 \mathrm{ng} / \mathrm{ml}$ recombinant murine GM-CSF (Peprotech Inc., Rocky Hill, NJ, USA), 20 ng/ml recombinant murine IL-4 (Peprotech) and $50 \mu \mathrm{M}$ 2-mercaptoethanol (ME). After 6 days of culture, non-adherent and loosely adherent cells were harvested, washed, and used for the experiments.

Flow cytometry. The method for flow cytometry analysis has been reported previously (25). PE-conjugated streptavidin,
mAbs against murine H-2D ${ }^{\mathrm{d}}$, CD4, CD11c, CD86, and FITCconjugated mAbs against murine $\mathrm{I}-\mathrm{A}^{\mathrm{d}}, \mathrm{CD} 3, \mathrm{CD} 8, \mathrm{CD} 54$ and CD80 were purchased from BD Pharmingen (San Diego, CA, USA). Biotin-conjugated mAbs against murine NK1.1, CD90.2 (Thy1.2), and Annexin-V were also purchased from BD Pharmingen. Cells incubated with anti-FcRII were stained as previously described with the appropriate mAb in PBS containing $2 \%$ FCS and $0.01 \%$ sodium azide. The stained cells were analyzed using a FACS Caliber flow cytometer (Becton-Dickinson, Mountain View, CA, USA) and the CellQuest software package (BD Biosciences). Necrotic cells and cellular debris, identified by forward scatter/side scatter (FSC/SSC) profiles and propidium iodide (PI; Sigma-Aldrich, St. Louis, MO, USA) staining, were excluded from the analysis.

Generation of CT26 tumor lysate. Soluble CT26 tumor lysate was obtained from subcutaneous CT26 tumors at the flanks of Balb/c mice. To generate these tumors, six-week-old female Balb/c mice were inoculated in the flank with $2 \times 10^{6}$ CT26 cells. Three weeks after inoculation, the excised tumors were homogenized at $4^{\circ} \mathrm{C}$ in $0.05 \mathrm{M}$ Tris- $\mathrm{HCl}(\mathrm{pH} 7.4)$ containing $0.5 \mathrm{M}$ sodium chloride, $5 \mathrm{mM}$-ethylmaleimide, $5 \mathrm{mM}$ EDTA, $1 \mathrm{mM}$ phenylmethyl-sulfonylfluoride, and antibiotics. The extracts were centrifuged at 50,000 x g for $30 \mathrm{~min}$ and the supernatants were dialyzed in phosphatebuffered saline. The concentration of tumor-lysate protein was determined by Lowry's method using the DC protein assay kit (Bio-Rad, Hercules, CA, USA).

Generation of stressed cells. CT26 cells were stressed in one of two ways in preparation for the antigen pulsing of DCs: Cells were suspended in PBS and either subjected to five cycles of rapid freezing and thawing, or exposed to $30 \mathrm{~Gy}$ irradiation. For the detection of apoptotic or necrotic cells, the stressed cells were first stained with annexin V-biotin according to the manufacturer's instructions and then stained with streptavidin-PE (BD Pharmingen) and PI. The stained cells were analyzed using flow cytometry.

DCs pulsed with tumor lysate or stressed tumor cells. Soluble tumor lysate was added to 6-day-old DC cultures at $500 \mathrm{mg}$ lysate $/ 1 \times 10^{6}$ cells in $1 \mathrm{ml}$ and then incubated at $37^{\circ} \mathrm{C}$ for $18 \mathrm{~h}$ in the presence of $50 \mathrm{mg} / \mathrm{ml}$ keyhole limpet hemocyanin (KLH; Carbiochem, Inc., La Jolla, CA, USA). Excess antigen was removed by centrifugation at $12,000 \mathrm{rpm}$ for $5 \mathrm{~min}$. The irradiated or freeze-thawed CT26 cells were cultured at a ratio of 1:1 with 6-day-old DC cells $\left(2 \times 10^{6} \mathrm{DC} / \mathrm{ml}\right)$ for $24 \mathrm{~h}$ at $37^{\circ} \mathrm{C}$ in the presence of $50 \mathrm{mg} / \mathrm{ml} \mathrm{KLH}$. The cells were then collected and prepared for vaccination as described for DCs pulsed with stressed CT26 cells.

Generation of DC/TC hybrids. Bone marrow-derived DCs were fused with irradiated tumor cells (30 Gy) at a ratio of $2: 1$, respectively, using a solution of $50 \%$ polyethylene glycol (m.w. 1450) in DMSO (Sigma-Aldrich), as described previously (26). Briefly, the DC/tumor-cell suspension was washed twice with RPMI-1640, and then resuspended in $1 \mathrm{ml}$ $50 \%$ polyethylene glycol added over $1 \mathrm{~min}$ and stirred gently for 1-2 min. Pre-warmed RPMI-1640 was then added slowly 
by gently stirring the suspension. After $2 \min$ at $37^{\circ} \mathrm{C}$, the resultant mixture of cells was grown overnight in a culture medium containing GM-CSF and $50 \mathrm{mg} / \mathrm{ml} \mathrm{KLH}$. In the present study, loosely adherent, DC/irradiated tumor-cell fusion hybrids were used for vaccination $24 \mathrm{~h}$ after chemical fusion.

To determine the efficiency of PEG-mediated fusion, DCs were stained with PE-conjugated murine anti-CD11c $\mathrm{mAb}$, whereas tumor cells were labeled with CFSE (Molecular Probes, Eugene, OR, USA) at a concentration of $10 \times 10^{6}$ cells $/ \mathrm{ml}$ in the presence of $5 \mathrm{mM}$ dye for $10 \mathrm{~min}$ at $37^{\circ} \mathrm{C}$ followed by the addition of ice-cold HBSS. After fusion procedures, those cells were analyzed by flow cytometry.

In vivo vaccination. Mice were immunized subcutaneously on the left flank with either $0.5-1 \times 10^{6}$ unpulsed DCs (DC only) as a control, DCs pulsed with tumor lysate (DC/lysate), DCs pulsed with freeze-thawed tumor cells (DC/F-T), DCs pulsed with irradiated tumor cells (DC/XRT), or DC and irradiated tumor cell fusion hybrids (DC/TC hybrid) in a volume of 0.1 $\mathrm{ml}$ at biweekly intervals on day 0 and 14 . One week after the final vaccination (day 21), the mice in each group were inoculated subcutaneously with varying doses $\left(1 \times 10^{4}, 10^{5}\right.$, $10^{6}$ cells/mouse) of viable CT26 cells. Three weeks after the tumor challenge (day 42), two perpendicular dimensions of each tumor were measured with a vernier caliper, and the tumor volume was calculated as the product of bisecting tumor diameters (long diameter $\mathrm{x}$ short diameter: $\mathrm{mm}^{2}$ ).

CTL assays. Procedures to measure CTL activity have been described previously (27). In brief, $3 \times 10^{6}$ splenocytes isolated 7 days after the last immunization (day 21) were cocultured with irradiated (30 Gy) $1 \times 10^{6}$ CT26 cells in 24-well culture plates for 5 days in RPMI/10\% FCS and $50 \mu \mathrm{M} 2-\mathrm{ME}$. After this restimulation, the splenocytes were collected and tested for their ability to lyse target cells in a standard 4-h ${ }^{51} \mathrm{Cr}$ release assay. The CTL activity of $\mathrm{CD} 4^{+}$and $\mathrm{CD}^{+} \mathrm{T}$ lymphocytes isolated from harvested splenocytes using magnetic cell-separation (MACS) CD90 (Thy1.2) beads (Miltenyi Biotec, Bergisch Gladbach, Germany) was tested relative to whole splenocytes. Effector (E) cells were added to $\mathrm{CT} 26$ target $(\mathrm{T})$ cells labeled with ${ }^{51} \mathrm{Cr}$ at various $\mathrm{E} / \mathrm{T}$ ratios (performed in triplicate). The percentage of cytotoxicity was calculated from the release of ${ }^{51} \mathrm{Cr}$ as follows: $100 \mathrm{x}$ [(experimental release) - (effector spontaneous release) (target spontaneous release)]/[(target maximum release) (target spontaneous release)]. Results are representative of five independent experiments.

Cytokine assays. Splenocytes $\left(3 \times 10^{6}\right.$ cells) obtained from immunized mice on day 21 were cultured with irradiated (30 Gy) CT26 cells ( $1 \times 10^{6}$ cells) in 24 -well plates for $48 \mathrm{~h}$ in RPMI/10\% FCS and $50 \mathrm{mM}$ 2-ME. After restimulation, the supernatants from these cultures were collected and IFN- $\gamma$ or IL-10 secretion from the splenocytes was measured using commercial ELISA kits (BD Pharmingen). Results from three independent experiments are expressed as mean $\pm \mathrm{SD}$.

Statistical analysis. The numerical data from the multiple trials are presented as mean \pm SD. Statistical analyses including the
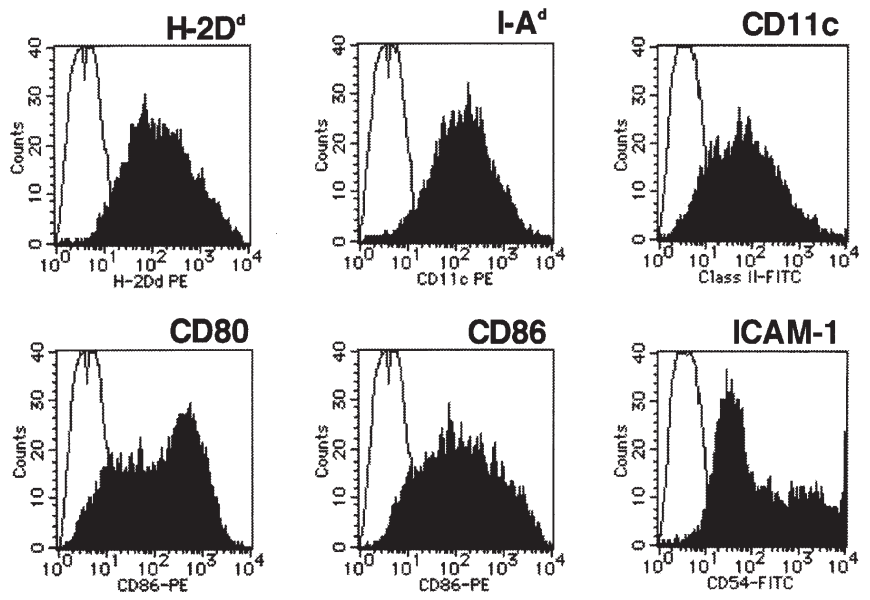

Figure 1. FACS profiles of day-6 bone marrow-derived DCs cultured in GM-CSF and IL-4. Cells demonstrate high levels of H-2D ${ }^{\mathrm{d}}$ (MHC class I), I-A ${ }^{\mathrm{d}}$ (MHC class II), CD11c, CD80, CD86 and ICAM-1.

Student's t-test, Mann-Whitney U test and Fisher's test were performed using Dr. SPSS II software (SPSS Japan Inc., Tokyo, Japan); P-values $<0.05$ were considered statistically significant.

\section{Results}

DC surface phenotype. DCs were prepared from bone marrow precursors and harvested for use after 6 days in culture media containing GM-CSF and IL-4. Before antigen pulsing, the DCs were stained with the appropriate FITC, PE or biotin-conjugated monoclonal antibodies for FACS analysis of the surface expression of MHC and costimulatory molecules. The DCs expressed high levels of the MHC class I molecule $\mathrm{H}-2 \mathrm{D}^{\mathrm{d}}$, the MHC class II molecule I-A ${ }^{\mathrm{d}}$, a DC marker CD11c, and costimulatory molecules CD80, CD86 and ICAM-1 (CD54) (Fig. 1). This pattern of surface molecules indicates that the bone marrow-derived DCs cultured for 6 days were nearing maturity. Changes in the levels of CD80, CD86, H-2D ${ }^{\mathrm{d}}, \mathrm{I}-\mathrm{A}^{\mathrm{d}}$ and CD54 were found to be insignificant following various strategies of Ag-loading the DCs (data not shown), suggesting that the antigenloading strategy has no effect on the maturation of DC surface molecules.

Extent of apoptotic vs necrotic tumor cells following various methods of stress. The tumor cells stressed as described above to induce apoptotic or necrotic tumor cells were analyzed using the Annexin-V/PI FACS assay. Annexin-V/PI double-positive cells were defined as necrotic, whereas cells positive for Annexin-V only were defined as apoptotic (28). The tumor cells treated by freeze-thaw exposure were predominantly positive for both Annexin-V and PI (Fig. 2A). However, irradiated cells were predominantly positive for Annexin-V only (Fig. 2B). Microscopic analysis of the tumor cells by trypan blue exclusion immediately after freeze-thaw revealed nearly $100 \%$ positive staining, whereas irradiated cells showed $>90 \%$ viability. Staining $24 \mathrm{~h}$ after each stress treatment demonstrated similar results. Despite the viability of irradiated tumor cells, the DC vaccines pulsed with 


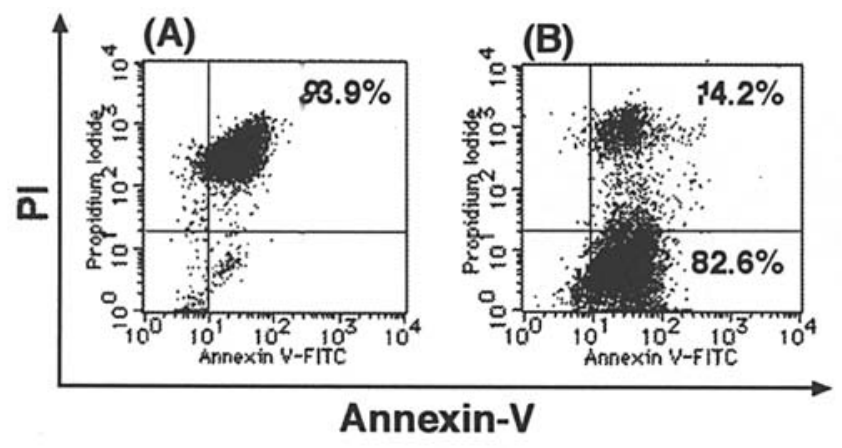

Figure 2. FACS profiles of stressed CT26 tumor cells for the antigen pulsing of DCs. (A) CT26 tumor cells suspended in PBS were subjected to five exposures to rapid freezing and thawing. Most of the cells (93.9\%) express both Annexin-V and PI, the phenotype of necrotic cells. (B) CT26 tumor cells exposed to $30 \mathrm{~Gy}$ irradiation predominantly express Annexin- $\mathrm{V}$ only $(82.6 \%)$, and the remainder express both Annexin-V and PI (14.6\%). Immediate microscopic analysis of both freeze-thawed tumor cells and $30 \mathrm{~Gy}$ irradiated tumor cells by trypan blue exclusion corresponded well with these FACS profiles (data not shown).

irradiated cells, as well as DC/irradiated cell hybrids, never resulted in tumor growth at the injection site. This result suggests that the cell death mechanism in irradiated tumor cells differs from freeze-thawed tumor cells, with irradiation inducing the apoptosis and exposure of cells to freeze-thaw leading to necrosis. Based on these findings, we concluded that 30 Gy irradiated tumor cells could be used safely as the source of tumor antigen.
Various antigen-loading strategies for DC-based vaccination. Table I lists the four strategies for antigen loading that were compared in this study: DC/lysate, DC/F-T, DC/XRT and $\mathrm{DC} / \mathrm{TC}$ hybrids were prepared as described. The number of CT26 cells that were used to prepare antigen-loaded DCs for one immunization was lowest in DC/TC hybrids. The fusion efficiency was calculated as the percentage of both CD11c and CFSE-stained cells in the FACS analysis (data not shown). The mean efficiency of the PEG-mediated fusion was $5.1 \%$ (range $3-15 \%$ ), which is lower than the efficiencies of $17-30 \%$ reported for the chemical fusion procedures.

Complete rejection induced by DCs pulsed with irradiated tumor cells and DC/tumor cell hybrids. To determine the in vivo anti-tumor protective effect of DCs pulsed with various antigen preparations, mice were divided into five groups and inoculated with $1 \times 10^{4}$ CT26 viable cells one week after the last immunization. Mice immunized with only DCs formed the control group. Three weeks after inoculation with the tumor cells, the mean and range (mean \pm SD) of the tumor volumes and the rejection rate were calculated for each group. For calculating the rejection rate, mice showing no tumor growth were defined as rejection mice (Table II). No mice immunized with DC only or DC/lysate rejected tumor growth, but the average size of tumors in mice immunized with DC/lysate was slightly smaller than tumors in mice immunized with DC only. Although the average tumor size in mice immunized with DC/F-T was significantly smaller than in mice immunized with DC only or DC/lysate, this group

Table I. Four different antigen loading strategies using whole tumor materials for DC-based vaccination.

\begin{tabular}{llc}
\hline Types of strategy & \multicolumn{1}{c}{ Method } & DC:TCa \\
\hline DC/lysate & DCs pulsed with soluble CT26 tumor cell lysate & $1: 2$ \\
DC/F-T & DCs pulsed with freeze-thawed CT26 tumor cells & $1: 1$ \\
DC/XRT & DCs pulsed with 30 Gy irradiated CT26 tumor cells & $1: 1$ \\
DC/TC hybrids & DC and 30 Gy irradiated CT26 tumor cell fusion hybrids & $2: 1$ \\
\hline
\end{tabular}

aThe ratio of DCs to CT26 tumor cells utilized for the production of soluble tumor lysate, the pulsing of DCs, or the generation of fusion hybrids with DCs.

Table II. The protective anti-tumor efficacy of mice immunized with four different antigen-loaded DCs.

\begin{tabular}{lccrr}
\hline Method of vaccination & No. of rejection & $\%^{\mathrm{a}}$ & Tumor size $^{\mathrm{b}}$ & Range $^{\text {a }}$ \\
\hline DC only & $0 / 5$ & 0 & $328.3 \pm 14.3$ & $310-360$ \\
DC/lysate & $0 / 6$ & 0 & $267 \pm 51.8$ & $218-345$ \\
DC/F-T & $2 / 4$ & 50 & $75 \pm 99^{c, d}$ & $80-220$ \\
DC/XRT & $11 / 11$ & 100 & - & - \\
DC/TC hybrids & $5 / 5$ & 100 & - & - \\
\hline
\end{tabular}

${ }^{a}$ The percentage of complete tumor rejection was calculated as 100x (the number of mice that completely rejected tumor growth)/(the total number of mice inoculated with $1 \times 10^{4} \mathrm{CT} 26$ cells in each of the groups), bthe product of bisecting the long and short diameter of the tumor 42 days after the last immunization $\left(\mathrm{mm}^{2}\right),{ }^{\mathrm{c}} \mathrm{P}<0.01$ (DC/F-T vs DC only), ${ }^{\mathrm{d}} \mathrm{P}<0.05$ (DC/F-T vs DC lysate). 


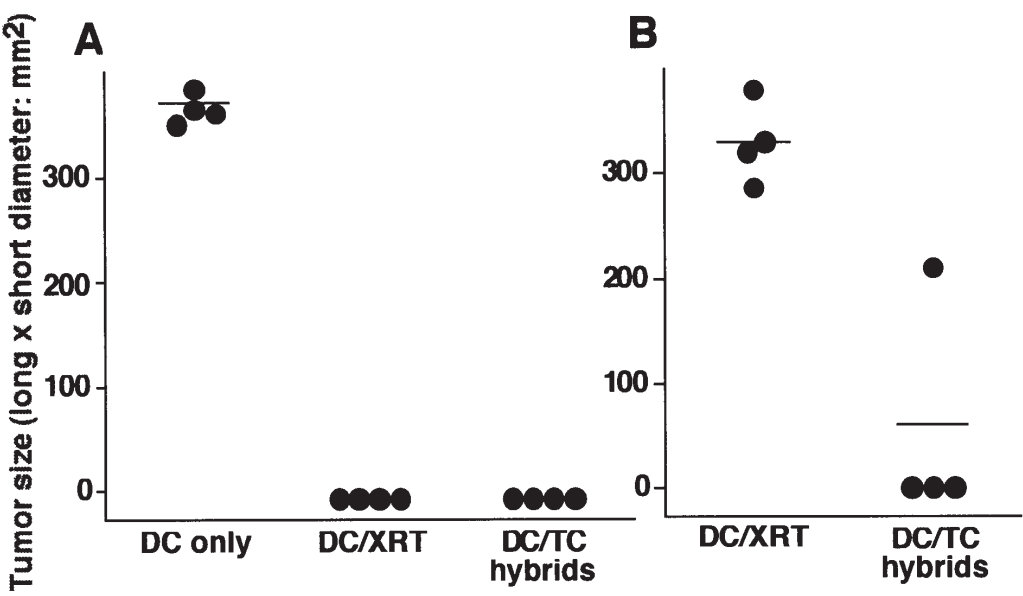

Figure 3. In vivo challenge test for anti-tumor protective immunity in mice immunized with DC only, DC/XRT or DC/TC hybrids. On day 0 and $14,0.5-1 \times 10^{6}$ unpulsed DCs, DCs pulsed with 30 Gy-irradiated CT26 tumor cells, or DC/CT26 tumor cell hybrids were injected subcutaneously into the left flank of each mouse. On day 21 , (A) $1 \times 10^{5}$ (B) $1 \times 10^{6}$ CT26 tumor cells were inoculated subcutaneously into the right part of the back, and on day 42 the size of each tumor was measured and its volume calculated from the product of bisecting long and short diameters ( $\mathrm{n}=4$ in each group). The level line shows the mean tumor volume for each group.

showed a $50 \%$ rejection rate. Notably, the rejection rate in both DC/XRT and DC/TC hybrids was $100 \%(11 / 11,5 / 5)$, and these mice showed no tumor growth throughout the extended observation period (data not shown). These results demonstrate clearly that the DC/XRT and DC/TC hybrids were more effective for antigen loading than the DC/lysate or DC/F-T. Our results also suggest that apoptotic tumor cells are a more efficient and potent antigen source for inducing DC-mediated anti-tumor protection than necrotic tumor cells.

Superiority of DC/TC hybrids in rejecting higher doses of inoculated CT26 cells. The number of inoculated CT26 cells was increased from $1 \times 10^{4}$ to $1 \times 10^{5}$ or $1 \times 10^{6}$ cells/mouse to compare the rejection rates of the DC/XRT or DC/TC hybrids. When mice immunized with either DC/XRT or DC/TC hybrids were challenged with $1 \times 10^{5}$ tumor cells, both groups demonstrated a $100 \%$ rejection rate (Fig. $3 \mathrm{~A}$ ). When inoculated with $1 \times 10^{6}$ tumor cells, $75 \%$ of the mice immunized with DC/TC hybrids rejected tumor growth (3/4), whereas there was no rejection of tumor growth in the DC/XRT-immunized mice (Fig. 3B). Three DC/TC hybrid-immunized mice remained tumor-free throughout the extended observation period. The results of these anti-tumor challenge tests suggest that tumor rejection with DC/TC hybrids was significantly more frequent and effective than with DC/XRT.

Excellent induction of CTLs in mice immunized with DC/TC hybrids. The greatest efficacy of anti-tumor protection was observed in mice immunized with DC/TC hybrids, followed by DC/XRT, DC/F-T and lastly, DC/lysate. To assess the anti-tumor efficacy of immunized mice in vitro, the CTL response was analyzed in the four groups using standard 4-h ${ }^{51} \mathrm{Cr}$ release cytotoxity assays. After restimulation by irradiated CT26 target cells, whole splenocytes were used to prepare effector cells. In accordance with the in vivo protective responses observed in the challenge tests, the splenocytes from mice immunized with DC/TC hybrids demonstrated the strongest CTL response (Fig. 4A). The CTL response of the splenocytes from mice immunized with DC/lysate was weak.
The CTL response of the splenocytes from mice immunized with DC/XRT or with DC/F-T was intermediate. As expected, the CTL response of the splenocytes from mice immunized with DC/XRT was somewhat stronger than that of mice immunized with DC/F-T. In additional experiments, Thy-1 $1^{+}$ splenocytes $\left(\mathrm{CD}^{+}{ }^{+}\right.$or $\mathrm{CD}^{+} \mathrm{T}$ lymphocytes) collected using magnetic cell separation (MACS) CD90 (Thy1.2) beads were used as effectors, with the same number of cells as the prior experiments with whole splenocytes. Mice immunized with DC/lysate showed no significant CTL response against CT26 target cells (Fig. 4B). The CTL responses from mice immunized with DC/XRT or DC/F-T were similar and weak relative to those from mice immunized with DC/TC hybrids. The CTL response of Thy- $1^{+}$splenocytes was inferior to that of whole splenocytes in mice immunized with DC/TC hybrids, DC/XRT or DC/F-T. These results demonstrate a positive correlation between the CTL response and antitumor protective efficacy. Results in vitro also suggest that effector cells other than $\mathrm{CD}^{+}$cytotoxic $\mathrm{T}$ lymphocytes that are present in whole splenocyte preparations (i.e. NK cells and NKT cells), and are likely to contribute synergistically with CTLs to splenocyte-mediated tumor-cell lysis activity.

High ratio of Th1- to Th2-type cytokines induced by DC/TC hybrid vaccines. To determine the effect of different antigenloading strategies on the balance between Th1 (IFN- $\gamma$ )- and Th2 (IL-10)-mediated responses, cytokines produced by splenocytes stimulated by various antigen-loaded DCs were analyzed. The concentration of IFN- $\gamma(\mathrm{pg} / \mathrm{ml})$ from DC/ lysate-immunized mouse splenocytes was half that of DC/F-T mice, and one-third that of DC/XRT-immunized mice. The highest concentration of IFN- $\gamma$ was produced by the splenocytes from mice immunized with DC/TC hybrids. In contrast, there were no significant differences between the concentrations of IL-10 produced by the splenocytes from mice immunized with various forms of antigen (Fig. 5A).The Th1/Th2 balance (Fig. 5B) is defined by the ratio of IFN- $\gamma$ concentration to IL-10 concentration. A bias towards more of the dominant Th1 cells may enhance the protective anti- 


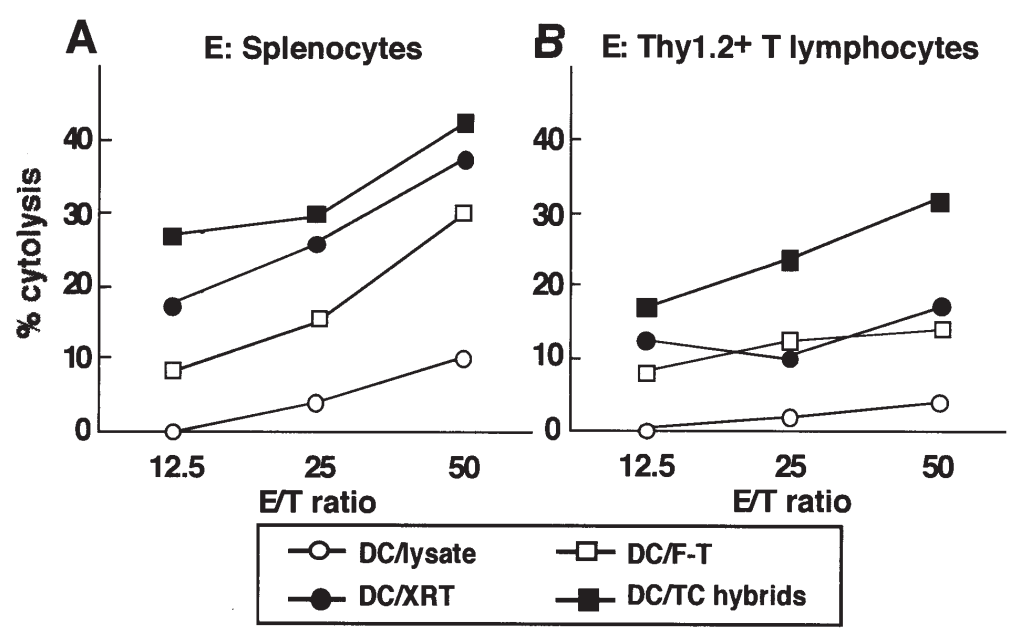

Figure 4. Induction of CTL responses in mice immunized with one of four different antigen-loaded DCs. Mice were immunized on day 0 and day 14 , and the splenocytes harvested on day 21 were stimulated with 30 Gy-irradiated CT26 tumor cells for 5 days. (A) After restimulation, the splenocytes from mice

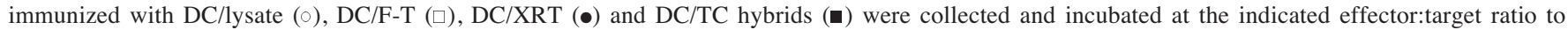
determine their ability to lyse CT26 tumor cells in a standard 4-h ${ }^{51} \mathrm{Cr}$ release assay. (B) T lymphocytes collected from the restimulated splenocytes isolated from mice immunized with DC/lysate, DC/F-T, DC/XRT, and DC/TC hybrids were tested for their ability to lyse target cells relative to whole splenocytes. For each assay, the percentage of effector cells was calculated from a total of $3 \times 10^{6}$ cells.

A
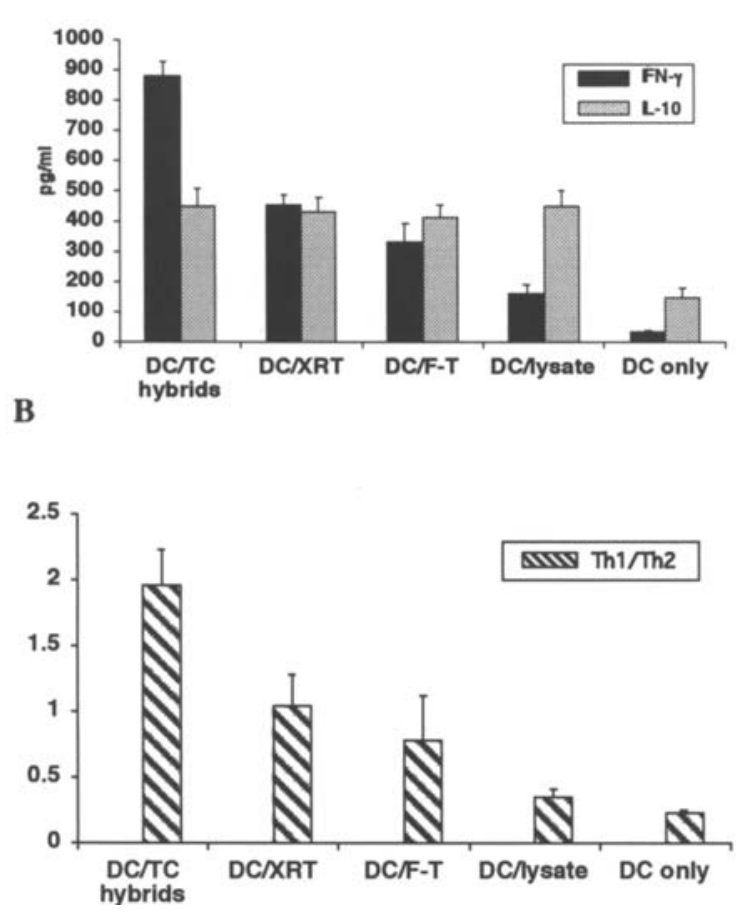

Figure 5. Cytokines produced from immunized splenocytes. Mice were immunized on day 0 and 14 with DC only or DCs pulsed with CT26-derived tumor antigen by four different strategies. The splenocytes harvested on day 21 were co-cultured with 30 Gy-irradiated CT26 tumor cells. (A) The supernatants from these cultures were collected and the secretion of IFN- $\gamma$ or IL-10 was measured by ELISA ( $n=5$ in each of the groups). (B) The ratio of IFN- $\gamma$ / IL-10 functioned as a measure of the Th1/Th2 balance for each group; bar, $\pm \mathrm{SD}$.

tumor immunity of each antigen-loading strategy. The Th1 to Th2 ratio of splenocytes from DC/TC hybrid-immunized mice was twice or three times higher, respectively, than that from mice immunized with DC/XRT or DC/F-T. Among the four methods, the Th1 to Th2 ratio in the splenocytes from DC/lysate-immunized mice was lowest, and was only twofold higher than that in DC-only-immunized control mice (Fig. 5B). In conclusion, DC/TC-hybrid vaccinated mice demonstrated the highest concentration of IFN- $\gamma$ produced by the splenocytes, as well as superior polarization of the Th1/ Th2 balance in favor of Th1.

\section{Discussion}

DCs pulsed with tumor Ags play a crucial role in anti-tumor immunity by inducing tumor-specific immune responses. However, a significant percentage of patients with advanced or metastatic tumors respond poorly to DC-based immunotherapy, as well as adjuvant therapy such as chemotherapy. To improve the efficacy of DC-based immunotherapies against cancer, numerous parameters need to be taken into account and optimized, including DC maturation, choice of Ag or Ag-loading method, injection site, timing and dosing, and appropriate anti-tumor immunological monitoring to measure the immune and clinical responses.

Whole tumor cells or their derivatives pulsed with DCs are commonly used tumor Ags in clinical immunotherapy trials. Unlike specific and known tumor-associated peptide antigens, whole tumor cells or their derivatives may better apply to a variety of tumors for which few or no defined tumor-specific antigens are available, such as colon cancer. Furthermore, the application of whole tumor cell immunizations can result in the polyvalent stimulation of both $\mathrm{CD}^{+}$ CTLs and CD4 ${ }^{+}$Th cells against a broad range of antigens. Among the current methods used to pulse DCs with whole tumor-derived materials, DC-tumor cell fusion hybrid vaccines have been suggested to be more potent in generating protective and therapeutic anti-tumor immunity $(19-21,29)$.

Similar to recent reports by Galea-Lauri et al $(19,21)$ and Kao et al (29), our tumor challenge tests demonstrated that DC-tumor cell fusion hybrids elicited the most significant 
degree of protection in mice challenged with high doses of tumor cells (Table II, Fig. 3). Additionally, irradiated apoptotic tumor cells activated a more potent anti-tumor response than freeze-thawed necrotic tumor cells in a murine colon cancer model. Finally, DCs pulsed with tumor lysate, although relatively more effective than DC-only controls, failed to elicit efficacious anti-tumor activity in vaccinated mice.

Certain studies suggest that the anti-tumor potency of different DC-based immunotherapies may be related to the mechanisms by which DCs capture, process, and present antigens, but not to the degree of DC maturation or the number of antigen-loaded DCs. Similar to other studies $(19,21)$, our experiments with DCs pulsed with tumor lysate failed to demonstrate efficacious anti-tumor protection or CTL response (Table II, Fig. 4). This failure may be explained by a model which hypothesizes that a majority of soluble tumor lysate is processed for MHC class II presentation, and rarely cross-presented by the MHC class I pathway, which theoretically promotes efficient CTL responses and antitumor effects (27). Some studies have demonstrated that DCs pulsed with tumor lysate are potent activators of anti-tumor immune responses in fibrosarcoma, mammary carcinoma and melanoma $(3,7)$. DCs pulsed with tumor lysate have been used in approximately one-third of recent DC-based vaccination immunotherapy clinical trials. Nonetheless, our results suggest that the immune response elicited by DCs pulsed with tumor lysate may not lead to sufficient antitumor potency in patients with colorectal cancer.

In contrast, it is likely that stressed tumor cells, including necrotic and apoptotic tumor cells, are captured and processed through both MHC class I and class II presentation pathways. Therefore, DCs pulsed with stressed tumor cells can prime tumor-specific CTLs and Th cells. In this study, tumor cells were stressed by one of two: Five cycles of freeze-thaw exposure led tumor cells to necrosis, whereas cells irradiated with 30 Gy died by apoptosis (Fig. 2). Data from anti-tumor challenge tests (Table II) suggest that apoptotic tumor cells are more potent tumor antigens for DC pulsing than the necrotic tumor cells. Furthermore, CTL activity is greater in whole splenocytes derived from mice immunized with DCs pulsed with apoptotic tumor cells relative to mice immunized with DCs pulsed with necrotic tumor cells (Fig. 4A). Apoptotic tumor cells may be superior antigens because they elicit higher levels of heat shock protein expression and greater production of IL-12 on pulsed DCs than necrotic tumor cells, which would lead to more potent activation of tumor-specific CD8 CTLs (28).

DCs pulsed with apoptotic tumor cells may also activate NK and NKT cells more effectively than DCs pulsed with necrotic tumor cells. In our experiments, there was no difference in the CTL activity of T lymphocytes purified from splenocytes derived from mice immunized with DCs and either apoptotic or necrotic cells (Fig. 4B). The superior CTL activity from mice vaccinated with DCs pulsed with apoptotic, rather than necrotic tumor cells, may be due to more active NK and NKT cells, which make up the majority of non-CD8 T-lymphocyte effector cells. A DC-NK interaction, in which DCs activate NK cells by the expression of IL-12 or IL-15, and activated NK cells stimulate DCs by the secretion of IFN- $\gamma$ (30), might also play an important role in the priming and stimulation of CTLs. A report by Kotera et al (28), in which the secretion of IL-12p70 by DCs pulsed with apoptotic cells was higher than DCs pulsed with necrotic cells, supports this hypothesis.

Among the cells tested in this study, DC-tumor cell fusion hybrids induced the highest level of anti-tumor protective immunity (Table II, Fig. 3). In a series of protective challenge tests, the CTL activities of whole splenocytes and of purified $\mathrm{T}$ lymphocytes from mice immunized with DC-tumor cell fusion hybrids also reflect the superiority of this anti-tumor response (Fig. 4). The potency of DC-tumor cell fusion hybrids may be due to high levels of peptide expressed on the fusion hybrids in the context of appropriate MHC class I molecules. Pre-existing MHC class-I presented tumor-cell antigens may function simultaneously with additionallypresented antigens on the DC. These additional antigens are transferred to the DC in the form of genetic materials such as DNA, mRNA and protein, and can be presented in the context of either MHC class I or class II molecules $(4,29)$. Additionally, our data suggest that DC-tumor cell fusion hybrids not only stimulate high levels of IFN- $\gamma$ from effector cells (Fig. 5A), but also induce the strongest shift to a Th1 response (Fig. 5C). We hypothesize that in mice vaccinated with DC-tumor cell fusion hybrids, Th1, DC1 and NK cells activate each other effectively via cytokines such as IL-12, IFN- $\gamma$, IL-2 and IL-15 (31). Such cross-activation between cells may be the basis for DC-tumor cell hybrids inducing the most potent anti-tumor protective immunity and the strongest CTL activity among the four different antigen-loading strategies examined.

Parameters that may require further adjustment to maximize the anti-tumor effect of DC-tumor cell fusion hybrids include the DC maturation state, fusion efficiency between DC and tumor cells, and the use of appropriate adjuvants. In this study, the foreign helper protein keyhole limpet hemocyanin (KLH) was used as an adjuvant in all Agloading strategies. DCs pulsed with KLH in addition to tumor peptides or tumor lysates are reported to stimulate KLH-specific CD4+ ${ }^{+}$Th cells, which lead to more efficient induction of antigen-specific CTLs via CD40-mediated secretion of IL-12 from DCs (32). But, it has not been elucidated that KLH could induce an effective ability to stimulate tumor-specific CTLs in vaccines with DCs pulsed with stressed tumor cells, as well as DC-tumor cell fusion hybrids, so other adjuvants such as IL-12 or IL-2 may show more promising anti-tumor efficacy.

In conclusion, this study clearly demonstrates that for a mouse colon cancer model, DC-tumor cell fusion hybrid vaccines elicit a more potent anti-tumor immune response than other antigen-loaded DCs, including DCs pulsed with tumor lysate, necrotic tumor cells, or apoptotic tumor cells. Our results also suggest that apoptotic tumor cells may result in the presentation of superior tumor antigens than necrotic tumor cells. In future clinical trials, further investigations of DC-tumor cell fusion hybrids will be required to achieve more effective anti-tumor protection and therapeutic efficacy. These should include the investigation of the failure of hybrids to migrate to draining lymph nodes, generation of a sufficiently large number of tumor cells from a solid tumor such as colon 
cancer, and the validity of allogeneic DCs as alternative fusion partners. Because procedures that utilize whole tumor materials but not defined single epitopes are likely to overcome the complexity of tumor cells and induce broader and more effective tumor-specific immune responses, intensive interest should be rendered to DC-tumor cell fusion hybrid vaccines.

\section{References}

1. Gallucci S, Lolkema M and Matzinger P: Natural adjuvants: endogenous activators of dendritic cells. Nat Med 5: 1249-1255, 1999.

2. Boczkowski D, Nair SK, Snyder D and Gilboa E: Dendritic cells pulsed with RNA are potent antigen-presenting cells in vitro and in vivo. J Exp Med 184: 465-472, 1996.

3. Fields RC, Shimizu K and Mule JJ: Murine dendritic cells pulsed with whole tumor lysates mediate potent antitumor immune responses in vitro and in vivo. Proc Natl Acad Sci USA 95: 9482-9487, 1998.

4. Gong J, Chen DS, Kashiwaba M and Kufe D: Induction of antitumor activity by immunization with fusions of dendritic and carcinoma cells. Nat Med 3: 558-561, 1997.

5. Mayordomo JI, Zorina T, Storkus WJ, Zitvogel L, Celluzzi C, Falo LD, Melief CJ, Ildstad ST, Kast WM, Deleo AB and Lotze MT: Bone-marrow-derived dendritic cells pulsed with synthetic tumor peptides elicit protective and therapeutic antitumor immunity. Nat Med 1: 1297-1302, 1995.

6. Hsu FJ, Benike C, Fagnoni F, Liles TM, Czerwinski D and Taidi B: Vaccination of patients with B-cell lymphoma using autologus antigen-pulsed dendritic cells. Nat Med 2: 52-58, 1996.

7. Nestle FO, Alijagic S, Gilliet M, Sun YS, Grabbe S, Dummer R, Burg G and Schadendorf D: Vaccination of melanoma patients with peptide- or tumor lysate-pulsed dendritic cells. Nat Med 4: 328-332, 1998.

8. Murphy GP, Tjoa BA, Simmons SJ, Jarisch J, Bowes VA and Ragde H: Infusion of dendritic cells pulsed with HLA-A2 specific prostate-specific membrane antigen peptides: a phase II prostate cancer vaccine trial involving patients with hormonerefractory metastatic disease. Prostate 38: 71-78, 1999.

9. Kugler A, Stuhler G, Walden P, Zoller G, Zobywalski A and Brossart P: Regression of human metastatic renal cell carcinoma after vaccination with tumor cell-dendritic cell hybrids. Nat Med 6: 332-336, 2000.

10. Banchereau J, Palucka AK, Dhodapkar M, Burkeholder S, Taquet N, Rolland A, Taquet S, Coquery S, Wittkowski K, Bhardwaj N, Piniero L, Steinman R and Fay J: Immune and clinical responses in patients with metastatic melanoma to CD34(+) progenitor-derived dendritic cell vaccine. Cancer Res 61: 6451-6458, 2001

11. Brossart P, Wirths S, Stuhler G, Reichardt VL, Kanz L and Brugger W: Induction of cytotoxic T-lymphocyte responses in vivo after vaccinations with peptide-pulsed dendritic cells. Blood 96: 3102-3108, 2000.

12. Pecher G, Spahn G, Schirrmann T, Kulbe H, Ziegner M, Schenk JA and Sandig V: Mucin gene (MUC1) transfer into human dendritic cells by cationic liposomes and recombinant adenovirus. Anticancer Res 21: 2591-2596, 2001.

13. Chaput N, Schartz NE, Andre F, Taieb J, Novault S, Bonnaventure P, Aubert N, Bemard J, Lemonnier F, Merad M, Adema G, Adams M, Ferrantini M, Carpentier AF, Escudier B, Tursz T, Angevin E and Zitvogel L: Exosomes as potent cellfree peptide-based vaccine. II. Exosomes in $\mathrm{CpG}$ adjuvants efficiently prime naive Tc1 lymphocytes leading to tumor rejection. J Immunol 172: 2137-2146, 2004.

14. Banchereau J, Ueno H, Dhodapkar M, Connolly J, Finholt JP, Klechevsky E, Blanck JP, Johnston DA, Palucka AK and Fay J: Immune and clinical outcomes in patients with stage IV melanoma vaccinated with peptide-pulsed dendritic cells derived from $\mathrm{CD} 34^{+}$progenitors and activated with Type I interferon. J Immunother Emphasis Tumor Immunol 28: 505-516, 2005.

15. Ashley DM, Faiola B, Nair S, Hale LP, Bigner DD and Gilboa E: Bone marrow-generated dendritic cells pulsed with tumor extracts or tumor RNA induce antitumor immunity against central nervous system tumors. J Exp Med 186: 1177-1182, 1997.
16. Berard F, Blanco P, Davoust J, Neidhart-Berard EM, NouriShirazi M, Taquet N, Rimoldi D, Cerottini J, Banchereaux J and Palucka AK: Cross-priming of naive CD8 T cells against melanoma antigens using dendritic cells loaded with killed allogeneic melanoma cells. J Exp Med 192: 1535-1544, 2000.

17. Nouri-Shirazi M, Banchereau J, Bell D, Burkeholder S, Kraus ET, Davoust J and Palucka KA: Dendritic cells capture killed tumor cells and present their antigens to elicit tumor-specific immune responses. J Immunol 165: 3797-3803, 2000.

18. Gong J, Nikrui N, Chen DS, Koido S, Wu ZK, Tanaka Y, Cannistra S, Avigan D and Kufe D: Fusions of human ovarian carcinoma cells with autologus or allogenic dendritic cells induce antitumor immunity. J Immunol 165: 1705-1711, 2000.

19. Galea-Lauri J, Wells JW, Darling D, Harrison P and Farzaneh F: Strategies for antigen choice and priming of dendritic cells influence the polarization and efficacy of antitumor T-cell responses in dendritic cell-based cancer vaccination. Cancer Immunol Immunother 53: 963-977, 2004.

20. Shimizu K, Kuriyama H, Kjaergaard J, Lee W, Tanaka H and Shu S: Comparative analysis of antigen loading strategies of dendritic cells for tumor immunotherapy. J Immunother Emphasis Tumor Immunol 27: 265-272, 2004.

21. Galea-Lauri J, Darling D, Mufti G, Harrison P and Farzaneh F: Eliciting cytotoxic T lymphocytes against acute myeloid leukemia-derived antigens: evaluation of dendritic cell-leukemia cell hybrids and other antigen-loading strategies for dendritic cell-based vaccination. Cancer Immunol Immunother 51: 299-310, 2002.

22. Trefzer U, Herberth G, Wohlan K, Milling A, Thiemann M, Sharav T, Sparbier K, Sterry W and Walden P: Tumourdendritic hybrid cell vaccination for the treatment of patients with malignant melanoma: immunological effects and clinical results. Vaccine 23: 2367-2373, 2005

23. Marten A, Renoth S, Heinicke T, Albers P, Pauli A, Mey U, Caspari R, Flieger D, Hanfland P, Von Ruecker A, EisHubinber AM, Muller S, Schwaner I, Lohmann U, Heylmann G, Sauerbruch T and Schmidt-Wolf IG: Allogeneic dendritic cells fused with tumor cells: preclinical results and outcome of a clinical phase I/II trial in patients with metastatic renal cell carcinoma. Hum Gene Ther 14: 483-494, 2003.

24. Lutz MB, Kukutsch N, Ogilvie ALJ, Rossner S, Koch F, Romani N and Schuler G: An advanced culture method for generating large quantities of highly pure dendritic cells from mouse bone marrow. J Immunol Methods 223: 77-92, 1999.

25. Harris N, Campbell C, Le Gros G and Ronchese F: Blockade of CD28/B7 co-stimulation by mCTLA4-Hgamma1 inhibits antigen-induced lung eosinophilia but not Th2 cell development or recruitment in the lung. Eur J Immunol 27: 155-161, 1997.

26. Wang J, Saffold S, Cao X, Krauss J and Chen W: Eliciting T cell immunity against poorly immunogenic tumors by immunization with dendritic cell-tumor fusion vaccines. J Immunol 161: 5516-5524, 1998.

27. Ferlazzo G, Semino C, Spaggiari GM, Meta M, Mingari MC and Melioli G: Dendritic cells efficiently cross-prime HLA class I-restricted cytolytic $\mathrm{T}$ lymphocytes when pulsed with both apoptotic and necrotic cells but not with soluble cell-derived lysates. Int Immunol 12: 1741-1747, 2000.

28. Kotera Y, Shimizu K and Mule JJ: Comparative analysis of necrotic and apoptotic tumor cells as a source of antigen(s) in dendritic cell-based immunization. Cancer Res 61: 8105-8109, 2001.

29. Kao JY, Zhang M, Chen CM and Chen JJ: Superior efficacy of dendritic cell-tumor fusion vaccine compared with tumor lysatepulsed dendritic cell vaccine in colon cancer. Immunol Lett 101: 154-159, 2005.

30. Ferlazzo G, Pack M, Thomas D, Paludan C, Schmid D, Strowig T, Bougras G, Muller WA, Moretta L and Munz C: Distinct roles of IL-12 and IL-15 in human natural killer cell activation by dendritic cells from secondary lymphoid organs. Proc Natl Acad Sci USA 101: 16606-16611, 2004.

31. Ruckert R, Brandt K, Bulanova E, Mirghomizadeh F, Paus R and Bulfone-Paus S: Dendritic cell-derived IL-15 controls the induction of CD8 T cell immune responses. Eur J Immunol 33: 3493-3503, 2003.

32. Millard AL, Ittelet D, Schooneman F and Bernard J: Dendritic cell KLH loading requirements for efficient $\mathrm{CD}^{+}{ }^{+} \mathrm{T}$-cell priming and help to peptide-specific cytotoxic T-cell response, in view of potential use in cancer vaccines. Vaccine 21: 869-876, 2003. 